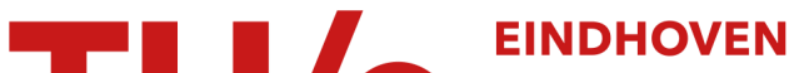 \\ UNIVERSITY OF \\ TECHNOLOGY
}

\section{Input/output selection for planar tensegrity models}

\section{Citation for published version (APA):}

Jager, de, A. G., \& Skelton, R. (2001). Input/output selection for planar tensegrity models. In Proceedings 40th IEEE Conference on Decision and Control (Orlando FL, USA, December 4-7, 2001) (pp. 4280-4285). Institute of Electrical and Electronics Engineers. https://doi.org/10.1109/.2001.980868

DOI:

$10.1109 / .2001 .980868$

Document status and date:

Published: 01/01/2001

\section{Document Version:}

Publisher's PDF, also known as Version of Record (includes final page, issue and volume numbers)

\section{Please check the document version of this publication:}

- A submitted manuscript is the version of the article upon submission and before peer-review. There can be important differences between the submitted version and the official published version of record. People interested in the research are advised to contact the author for the final version of the publication, or visit the $\mathrm{DOI}$ to the publisher's website.

- The final author version and the galley proof are versions of the publication after peer review.

- The final published version features the final layout of the paper including the volume, issue and page numbers.

Link to publication

\section{General rights}

Copyright and moral rights for the publications made accessible in the public portal are retained by the authors and/or other copyright owners and it is a condition of accessing publications that users recognise and abide by the legal requirements associated with these rights.

- Users may download and print one copy of any publication from the public portal for the purpose of private study or research.

- You may not further distribute the material or use it for any profit-making activity or commercial gain

- You may freely distribute the URL identifying the publication in the public portal.

If the publication is distributed under the terms of Article 25fa of the Dutch Copyright Act, indicated by the "Taverne" license above, please follow below link for the End User Agreement:

www.tue.nl/taverne

Take down policy

If you believe that this document breaches copyright please contact us at:

openaccess@tue.nl

providing details and we will investigate your claim. 


\title{
Input/output selection for planar tensegrity models
}

\author{
Bram de Jager \\ Department of Mechanical Engineering \\ Eindhoven University of Technology \\ P.O. Box 513, $5600 \mathrm{MB}$ Eindhoven, The Netherlands \\ Email: A.G.de.Jager@wfw.wtb.tue.nl Fax: +31 402461418 \\ Robert Skelton \\ Department of Mechanical and Aerospace Engineering \\ University of California at San Diego \\ La Jolla, California, 92093-0411 \\ Email: bobskelton@ucsd.edu Fax: +1 619/534-7078
}

\begin{abstract}
A systematic method of selecting sensors and actuators is produced, efficiently selecting inputs and outputs that guarantee a desired level of performance in the $\mathcal{H}_{\infty}$-norm sense. The method employs an efficiently computable necessary and sufficient existence condition, using an effective search strategy. The search strategy is based on a method to generate all so-called minimal dependent sets. This method is applied to tensegrity structures. Tensegrity structures are a prime example for application of techniques that address structural problems, because they offer a lot of flexibility in choosing actuators/sensors and in choosing their mechanical structure. The selection method is demonstrated with results for a 3 stage planar tensegrity structure where all 26 tendons can be used as control device, be it actuator, sensor, or both, making up 52 devices from which to choose. In our set-up it is easy to require devices to be selected as colocated pairs, and to analyze the performance penalty associated with this restriction. Two performance criteria were explored, one is related to the dynamical stiffness of the structure, the other to vibration isolation. The optimal combinations of sensors and actuators depend on the design specifications and are really different for both performance criteria.
\end{abstract}

Keywords: mechanical systems, tensegrity structures, $\mathcal{H}_{\infty}$-control, inputoutput selection, combinatorial optimization, maximal independent set, minimal dependent set

\section{Introduction}

The ultimately achievable performance of a controlled plant depends on plant characteristics, on controller architecture, and on controller tuning. Normally plant and controller are designed separately, which may lead to a suboptimal performance of the closed loop. Concurrently designing plant and control, an integrated design, is therefore important. An interesting topic is the selection of sensors and actuators, because they define the interface between plant and controller. Here, issues are the type, number, and place of devices for actuation and sensing or, more generally, of input and output signals used for the closed loop. Inappropriate selection of sensors may, for instance, lead to zeros in the right-half-plane, a well known performance limiting factor. Other limitations are a high relative degree, unmatched disturbance/model error inputs, and a large model uncertainty near cross-over. Therefore, one aims at selecting an appropriate controller structure, e.g., those input/output devices for which a controller exists that will deliver a desired level of performance, which will exclude combinations with performance limitations. Besides perfor- mance, also considerations like complexity and cost should be considered.

A prime application of integrated design techniques is tensegrity structures. These are web-like mechanical structures that consist of two types of elements: tensile members (tendons) and compressive members (bars) [1]. This class of systems has been studied for a long time, see, e.g., [2], whose terminology consisted of ties and struts instead of tendons and bars. In a class 1 tensegrity structure the bar endpoints, or nodal points, are only connected to tendons, not to other bars. Tendons are exclusively loaded in tension, otherwise they would buckle because they are very slender. Bars are normally loaded in compression only and not in tension. The integrity (stability) of a tensegrity structure is due to the tensile forces in the tendons, hence the name tensegrity.

Tendons in tensegrity structures have multiple roles, they:

- rigidize and stiffen the structure (pretension),

- carry structural loads,

- provide opportunities for actuation/sensing [3]

Actuation can improve properties like stiffness or stiffness-tomass ratio and damping. Sensing provides information about the geometry of the structure, the deformations, and the like. Actuation can be carried out by changing the length of the tendons or the bars. This can be done in several ways, by:

- shape memory alloys that enable the tendons to shorten and lengthen by changes in temperature,

- linear or rotary motors that can shorten a tendon by hauling it, e.g., inside hollow bars,

- extensible bars.

A target area of application for tensegrity structures is where the shape of a structure needs to be changed dynamically, e.g., in space technology with deployable structures or in medicine with expandable inserts.

Here we consider only the tendons as elements that can sense their own length and can change that length. Changing the unstressed length of tendons also changes the shape of the structure or eliminates deformations that occur due to external loads. The sensed information can be used in a control loop to send appropriate signals to the actuation system.

Due to the large number of possibilities to assign actuators and sensors, tensegrity structures need an efficient method for input/output selection. Solutions to input/output selection are abundant, for an overview see [4]. Selection of the devices or signals based on a full candidate-by-candidate feasibility test is a combinatorial problem. The selection can be simplified 
by not using a candidate-by-candidate approach, but then it is likely to be less effective and favorable combinations of actuators and sensors can be missed. Finding a good method for input/output selection is like a balancing act. Aiming at generality and rigor makes it quite hard $(\mathcal{N} \mathcal{P}$-hard, to be precise, see [5]) to find a solution, and therefore hardly practical for large systems. Making it easy to find an answer may lead to sloppy results, which cannot really be trusted or are not very specific. A selection using a single shot approach is possible, but only with a crude selection criterion or with an approximate solution.

In previous work [6], we applied an approach that is more refined than brute force methods. The selection is still based on a candidate-by-candidate like test. It uses a streamlined rigorous feasibility test combined with an efficient search strategy, but needs an additional, in general easily fulfilled, assumption. Large scale problems may then be tackled in acceptable time, because only a limited number of combinations need to be tested. The search strategy is based on an efficient implementation of an algorithm to generate all maximal independent sets (or minimal dependent sets) [7], which is a standard problem in combinatorial optimization. Although with this approach the problem is theoretically still combinatorial in the number of devices, in practice the complexity is affine in the number of inputs and outputs and in the size of the solution.

We consider a 3 stage planar tensegrity structure with 26 tendons and two different closed loop specifications. Available computer hardware did not allow for the selection from 52 devices simultaneously, so only 32 devices are allowed. To accommodate this restriction, the selection was split in three steps. First selecting actuators, assuming all sensors to be used. Then selecting sensors, assuming all actuators to be used. From the most promising results of these selections, a combination of 32 devices is selected to find solutions with a lower number of devices that still meets the required performance level. This is performed for several performance levels, to get insight in the relation between the number of devices and the achievable performance. This procedure is repeated for both design specifications. Alternatively, a problem with colocated sensor/actuator pairs, giving only 26 independent devices, has been the starting point for the, now much easier, selection.

The goal of the paper is to address the problem of efficient and effective input/output selection for planar tensegrity structures. The results depend on the design criteria, can be explained qualitatively, and make sense physically.

The paper is structured as follows. First, we discuss tensegrity structures and a dynamic model of planar tensegrity. Then we explain the search strategy and feasibility tests, and show how these methods can be applied on a large scale selection problem. Conclusions finish the paper.

\section{Planar tensegrity structures}

A tensegrity structure consists of bars and tendons, arranged in such a way that the structure has integrity and is not a mechanism. This is achieved by pre-stressing the tendons by a tensile force. A planar tensegrity structure is one that only extends in the plane. A tensegrity structure can be of class 1 , where bars are only connected to tendons, or of class 2 , where a connection can connect up to two bars and a number of tendons. This can be generalized to a class $k$ definition. Often a tensegrity structure is made up of nested tensegrity structures,

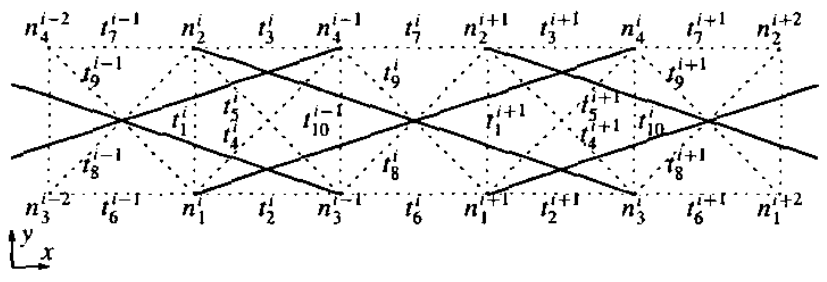

Figure 1: Single stage of planar tensegrity structure. Bars: tendons: - -

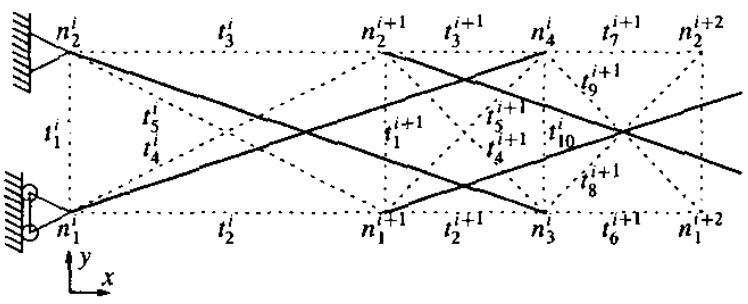

Figure 2: Left stage of planar tensegrity structure, $i=1$

giving it a fractal character. This is beneficial for analysis and design, because only a limited number of structures needs to be investigated. Those structures can then be used to build up a more complex structure.

An elementary stage, numbered $i$, of a planar tensegrity structure of class 1 is given in Fig. 1. This stage can be repeated indefinitely, by replicating it, shifted some distance of the horizontal dimension, to build up a planar structure in $x$-direction. It could also be replicated in $y$-direction or both.

Indicated are the numbering of the tendons that belong to stage $i$, given by $t_{\alpha}^{i}$, with $1 \leq \alpha \leq 10$. Also indicated are tendons of stages $i-1$ and $i+1$ that are connected to the four endpoints (nodes) $n_{1-4}^{i}$ of the two bars of stage $i$. Note that the number of tendons is not minimal. For instance, all diagonal tendons $t_{4,5.8 .9}^{i}$ can be removed, while the structure still has integrity and does not become a mechanism. Diagonal tendons are included because it avoids infinitesimal movements of the stages relative to each other without causing infinitesimal correcting forces. Without diagonal tendons the stiffness is derived from second order effects (i.e., it is zero in the linear approximation, except for pre-stress). So, a better approach to get a minimal number of tendons is to eliminate vertical tendons and keep some diagonal tendons.

The left side of the structure has to be modified, and is given in Fig. 2. Besides modification for the differences in boundary geometry, the left side removes the three degrees-of-freedom of the rigid body, in effect, it restricts movement of the upper left node in both $x$ and $y$-coordinate direction, i.e., the node is translationally fixed, and of the lower left node in the $x$ direction. A result of the restrictions is that the vertical left tendon $t_{1}^{1}$ of the structure cannot rotate, although both bars of stage $i=1$ are still free to rotate. Note that tendons $t_{6-9}^{i}$ do no longer appear for $i=1$ and that some tendons connect to other nodes than in the previous figure.

The right side is in Fig. 3. There are no restrictions specified at this boundary. Only differences in geometry are taken into account, the connection of some tendons is to different nodes than in Fig. 1. 


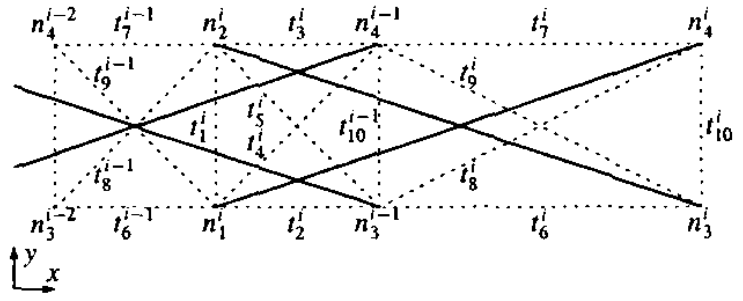

Figure 3: Right stage of planar tensegrity structure, $i=n$

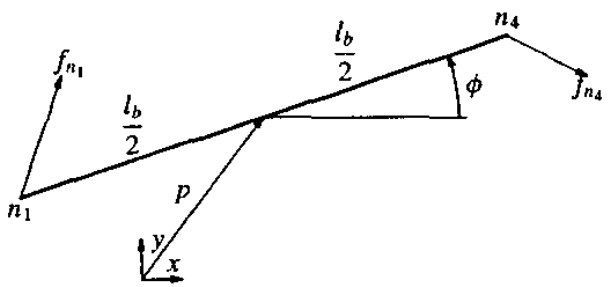

Figure 4: Elementary bar in planar tensegrity structure

\section{Tensegrity structure models}

Two models are developed, a nonlinear model for arbitrary displacements and a linearized model, valid for small displacements only, for use in a linear plant model. The nonlinear model can be used to evaluate the results with simulations, and to get access to robustness issues.

The basic assumptions in setting up the nonlinear model are

1. a bar is straight and of uniform cross section and density,

2. the central moment of inertia of a bar for rotation around its principle axis is zero,

3. a bar is of fixed length, so infinitely stiff axially,

4. a tendon is massless,

5. a tendon has no torsional or bending stiffness, but axial stiffness,

6. a bar has two nodal points, which are of zero dimension,

7. a tendon is connected to a bar at a nodal point only,

8. external loads are only applied at a nodal point,

9. external loads do not include bending or torsional loads,

10. there are no potential fields (e.g., gravity).

Due to these assumptions, the bars are axially loaded only, except during transients. Although elements in a tensegrity structure are axially loaded only, the structure itself has a finite stiffness for bending and torsion.

The model of the complete structure is quite elementary, being built up of bars that are connected by elastic tendons, and can best be developed by a classical Newtonian formulation, because we are also interested in forces internal to the structure.

The model for a single bar, see Fig. 4, moving in the plane is

$$
\begin{aligned}
m \ddot{p} & =F_{b} \\
J \ddot{\phi} & =M_{b}
\end{aligned}
$$

using as the three generalized coordinates the position $p$ of the center of mass and the orientation angle $\phi$ around this center. The mass $m$ and central moment of inertia $J$ are the physical parameters that specify the dynamics of the bar.

We can compute the forces $F_{b}$ and moment $M_{b}$ from the nodal force vectors $f_{n_{1}}$ and $f_{n_{A}}$, assumed given in Cartesian components, by

$$
\begin{aligned}
F_{b} & =f_{n_{1}}+f_{n_{4}} \\
M_{b} & =\frac{l_{b}}{2}\left[\begin{array}{ll}
\sin \phi & -\cos \phi
\end{array}\right] f_{n_{1}}+\frac{l_{b}}{2}\left[\begin{array}{ll}
-\sin \phi & \cos \phi
\end{array}\right] f_{n_{4}} .
\end{aligned}
$$

The model for a tendon can be derived from classical continuum mechanics. A simple model, linear elastic, for material behavior is $\sigma=E \varepsilon$ with $E$ the modulus of elasticity, and where $\sigma=F / A$, the stress, is the ratio of force and crosssectional area, and $\varepsilon=\Delta l / l_{0}$, the strain, is the elongation $\Delta l=l-l_{0}$ divided by the unstressed length $l_{0}$. This gives

$$
F=\frac{E A}{l_{0}}\left(l-l_{0}\right)=k_{t}\left(l-l_{0}\right)
$$

to compute the force $F$ given $l_{0}$ and $l$. To compute the unstressed length when both $F$ and $l$ are known,

$$
l_{0}=\frac{l}{1+\frac{F}{E A}} .
$$

Note that the length $l$ can be computed as the Euclidean norm of a tendon vector $t, l=\|t\|$.

A tendon vector $t$ is computed as the difference of the two nodal point vectors that the tendon connects to, and taken to point in up/right direction, where right takes precedence, $t=p_{n_{j}}-p_{n_{k}}$.

The Cartesian coordinates $p_{n}$ of the nodal points can be computed as

$$
p_{n_{1}}=p-\frac{l_{b}}{2}\left[\begin{array}{c}
\cos \phi \\
\sin \phi
\end{array}\right], \quad p_{n_{4}}=p+\frac{l_{b}}{2}\left[\begin{array}{c}
\cos \phi \\
\sin \phi
\end{array}\right] .
$$

The stressed length $l$ determines the tendon force magnitude $F$. The direction of the tendon force vector $f_{\text {, comes }}$ from the tendon vector $t$ because those vectors are aligned

$$
f_{t}=\frac{F}{\|t\|} t
$$

where the tendon vector needs to be scaled by its Euclidean norm.

Nodal forces $f_{n}$ are computed by summing tendon forces $f_{t}$ for those tendons connected to a particular node, taking account of the sign convention,

$$
f_{n}=\sum \pm f_{t}+w_{n}
$$

where $w_{n}$ is a load acting on nodal points.

The equations for individual bars can be taken together to form the following differential equations

$$
\begin{aligned}
\ddot{q} & =T(q, w) \\
q^{T} & =\left[\begin{array}{lllll}
p_{1 x} & p_{1 y} & \phi_{1} & \ldots & \phi_{2 n}
\end{array}\right]
\end{aligned}
$$

where $q$ gathers the positions $p$ and orientations $\phi$, the generalized force $T$ gathers the forces $F_{b}$ and moments $M_{b}$, and the load $w$ gathers $w_{n}$. For a static model $\ddot{q}$ is equated to zero and the resulting algebraic equations, $T(q, w)=0$, represent the equilibrium conditions.

The linearized model is obtained by taking finite differences around an equilibrium. The exogenous inputs are measurement noise and the external loads $w$. The to-be-controlled variables are displacements or accelerations of designated nodes, $p_{n}$ or $\ddot{p}_{n}$, and control inputs $u$. 


\section{The IO selection method}

We address the selection of actuating/sensing tendons that are useful to achieve a desired level of closed-loop performance. The performance criterion is based on the $\mathcal{H}_{\infty}$-norm of a closed-loop generalized linear system. Design specifications are embodied in selected weighting filters.

The goal is to characterize the full set of feasible solutions, i.e., combinations of actuators and sensors for which a controller exists that can guarantee the desired level of performance for the closed-loop system. Now an $\mathcal{N} \mathcal{P}$-hard problem, the maximal independent set problem, has to be solved.

To select combinations of inputs and outputs (also called IO sets), we need two things: an algorithm to efficiently search for promising combinations and a feasibility test that assesses a single candidate IO set. The feasibility test should be efficient because it is called often. The test we employ should tell something about control relevant performance. The remainder of this section addresses the following points

- Strategy for taming the combinatorially explosive search.

- A simple approach to circumvent time-consuming steps in the feasibility test.

\subsection{Search strategy}

The search strategy is based on an algorithm to generate all maximal independent or all minimal dependent sets. The algorithm was proposed in [5] and implemented in [7]. We briefly explain the problem setup and the usefulness of the algorithm. Let $E$ be the finite set of all sensors and actuators that are considered, with cardinality $|E|=n$, and let $\mathcal{I}$ be a nonempty family of subsets of $E$ that satisfies the following rule: if $I \in \mathcal{I}$ and $I^{\prime} \subseteq I$ then $l^{\prime} \in \mathcal{I}$. Now, $(E, \mathcal{I})$ is called an independence system and $\mathcal{I}$ is its family of independent sets. An independent set $I$ is called maximal if there is no $I^{\prime} \in \mathcal{I}$ such that $I^{\prime} \supset I$. Subsets of $E$ that are not in $\mathcal{I}$ are dependent sets. All dependent sets form the family $\mathcal{J}$. A dependent set $J$ is minimal if $J^{\prime} \in \mathcal{I}$ for all $J^{\prime} \subset J$.

The IO selection problem with a monotonous selection criterion exactly fits an independence system problem. A monotonous selection criterion is one where the performance always improves, or stays the same, when an IO set is expanded with additional devices. The family of subsets $\mathcal{I}$ gathers all actuator/sensor combinations that are not acceptable and $\mathcal{J}$ characterizes all acceptable ones. The power set $P(E)$ contains all possible combinations of actuators and sensors and $P=\mathcal{I} \cup \mathcal{J}$. The sets can be graphically represented in a so-called Hasse diagram.

Now the problem is to establish the structure of the independence system, i.e., to find $\mathcal{I}$ and/or $\mathcal{J}$. To do this, an oracle is available that decides whether a subset of $E$ belongs to $\mathcal{I}$ or $\mathcal{J}$. The oracle is expensive and its visits should be minimized. In general, it suffices to find the $K$ maximal independent sets of $\mathcal{I}$ or the $M$ minimal dependent sets of $\mathcal{J}$, because with these sets one can generate the families $\mathcal{I}$ and/or $\mathcal{J}$ without visiting the oracle. Because both $K$ and $M$ are bounded by $\left(\begin{array}{c}n \\ n / 2\end{array}\right)$, one cannot guarantee to obtain a solution in time polynomial in $n$. One may wonder if a solution in time polynomial in $n$ and $K$ or $M$ is possible: Lawler et al. [8] state that the problem of finding the $K$ maximal independent or $M$ minimal dependent sets is $\mathcal{N} \mathcal{P}$-hard and there is no solution possible in time polynomial in $n, K$, and $M$. However, in [5] it is shown to be possible to establish all $K$ maximal independent sets and all $M$ minimal dependent sets visiting the oracle only $O(n K+M)$ or $O(K+n M)$ times. This means that a complete solution with visits affine in $n, K$, and $M$ is possible. An algorithm that achieves this has been used.

When the number of possible devices is large, also the number of feasibility tests is large, because in general $M$ and $K$ will be large, except in those cases were either a very small or a very large fraction of the devices is needed to meet the performance level. In general, when $n>\approx 32$ one needs to consider alternative strategies. A possibility is to extract from separate input and output selection, with a smaller number of devices for each, those devices that are most promising, e.g., by selecting those that

- occur often in minimal dependent sets,

- occur in minimal dependent sets with a low number of devices,

- occur in maximal independent sets with a low number of devices,

- do not occur in maximal independent sets with a large number of devices.

By eliminating devices that are not expected to add much, the size of the problem is reduced and a combined selection is tractable. The application section gives examples of the use of these heuristic rules.

\subsection{Feasibility test}

The selection of IO sets with guaranteed performance is based on existence conditions for controllers that achieve a specified $\mathcal{H}_{\infty}$ performance bound. There are other methods that could be considered, like linear matrix inequalities (LMI). $\mathcal{H}_{\infty}$-techniques have the advantages of a sound theoretical foundation, of readily available analysis and synthesis software, and of necessary and sufficient existence conditions. The feasibility test consists of checking conditions for the existence of an $\mathcal{H}_{\infty}$ controller achieving a specified performance level. Efficient tools for this task are available and may be based on Riccati equations [9] or on conditions expressed in terms of LMI $[10,11]$. We employ Riccati equations, being more efficient.

The feasibility test consists of several necessary conditions, that together are sufficient. This leads itself naturally to a streamlined computation. If one of the necessary conditions is not fulfilled, the remaining conditions do not need to be checked. So only for feasible combinations all checks are done. By using a pre-set performance level, the $\gamma$-iteration usually employed in $\mathcal{H}_{\infty}$ designs is not needed.

\section{Application}

The selection method is illustrated for a 3 stage planar tensegrity model with twenty-six actuators and twenty-six sensors, so with $n=52$ input/output devices, making $\approx 2^{52}$ or $\approx 4.5 \cdot 10^{15}$ unique combinations possible. This is much larger than any other application of rigorous techniques considered before.

Two typical design specifications are explored. The first is to stiffen a planar tensegrity structure, shaped like a cantilever beam, for external loads. The second is to dampen vibrations when the structure is considered as an erected building loaded by ground excitations. Results are therefore presented for the following two cases:

1. dynamic stiffness improvement

2. vibration reduction.

For both cases the standard plant setup, using four types of signals (exogenous signals, controller inputs, to-be-controlled 
variables and measurements), is selected for our purposes, because it is general and embraces a lot of control problems, like setpoint regulation, tracking, and disturbance rejection, all in the face of model errors. The feasibility conditions are placed on a generalized linear plant that depends on the controller inputs and outputs.

To simplify matters, the weighting functions are chosen to be static weights. The weights are chosen so all weighted signals (measurement noise, external load, control input, displacement or acceleration) have an appreciable influence on the achievable $\mathcal{H}_{\infty}$-norm. Now, the number of states of the generalized plant is not that large, namely 36 , to speed up computations.

To use the independence system setup, noise present in the input signal should vanish if the signal's amplitude is zero.

\subsection{Dynamic stiffness improvement}

For the full IO set the achievable value of the $\mathcal{H}_{\infty}$-norm is slightly smaller than $\gamma=0.3$. For a required performance level of $\gamma=0.3$, IO selection has been carried out for a subset of 16 from the 26 tendons, so $n=32$, and the number of sensors and actuators in the base set is equal and allows colocation. The tendons selected were those that during separate input and output selection often yielded promising actuators or sensors. Giving results in a Hasse diagram does not make sense, due to the large number of IO sets, that need more pixels than available on a sheet of paper. So, a more condensed representation is chosen that only sums the number of occurrences of a device in the minimal dependent sets. Figure 5 is therefore a compact representation of the 40960 minimal dependent sets that completely characterize the feasible and infeasible 10 sets. There are four devices (2-5) that are always needed. Note that more actuators (devices numbered

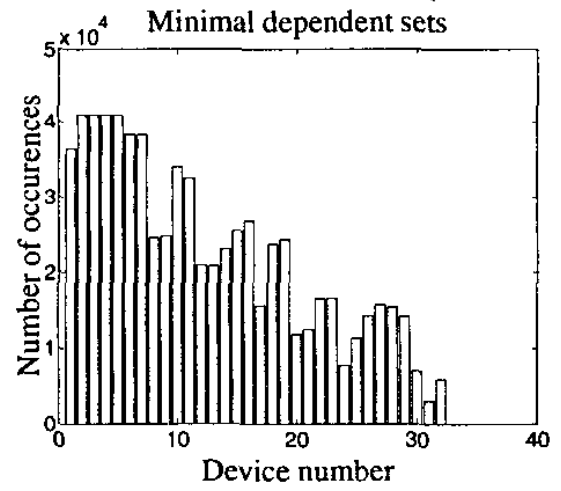

Figure 5: Results for minimal dependent IO sets for $\gamma=0.3$ 1-16) than sensors are needed, so not only colocated devices are selected.

Another way to present the results is by showing how many devices are in the minimal dependent or maximal independent sets. This information is in Fig. 6. The smallest feasible IO set has 14 devices, and there are 545 of these sets, mostly permutations of a slightly larger number of devices.

The most useful information is now available from the data for the maximal independent IO sets. Two of those sets have 13 devices and adding any of the remaining 19 devices makes them feasible, so the devices in those two sets are ranked high. There are 4 maximal independent sets of size $n-1$, which implies that 4 of the devices appear in all minimal dependent sets, because they are always needed, and rank therefore high.

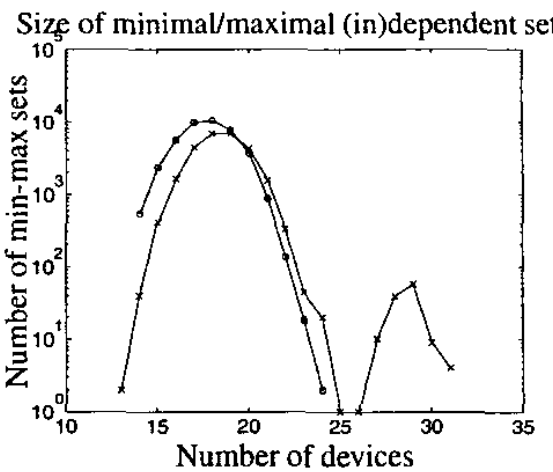

Figure 6: Number of devices for $\gamma=0.3$; "o":minimal dependent, " $x$ ": maximal independent 10 sets

This agrees with Fig. 5. A similar reasoning will prefer devices that are not included in maximal independents sets of size $n-2$. In this way also the most promising devices in separate input and output selection were chosen.

A physical interpretation of the results indicates that horizontal tendons, those "perpendicular" to the disturbance, are preferred, both for actuation and sensing. Not all selected IO sets were colocated ones.

\subsection{Vibration reduction}

For the full IO set the achievable value of the $\mathcal{H}_{\infty}$-norm is slightly smaller than 1 , namely $\gamma=0.9849$. Here we first present results for actuator selection (so $n=26$ ) that show how the search for the minimal dependent or maximal independent sets is accomplished. For a perfornance level $\gamma=5$, $M$ and $K$ are rather small, permitting presenting those results. The main purpose is to show how the search direction influences the number of feasibility tests to be performed.

Figure 7 presents results for finding minimal dependent sets, using a top-down search direction. The figure illustrates the

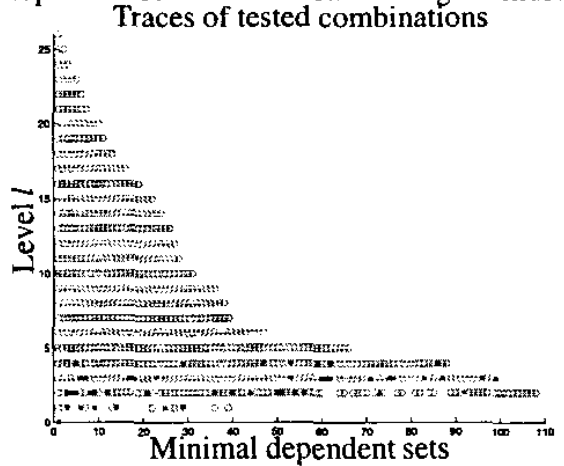

Figure 7: Search for minimal dependent inputs sets for $\gamma=5$; "o": feasible one, " $x$ ": infeasible ones

depth first search strategy. Starting from the top (with $l=$ $n=26$ ), devices from the full IO set are eliminated, until no further elimination is possible without becoming infeasible. Then another base IO set is chosen, with $l=n-1$ devices or with another permutation of devices, and the elimination starts again, until for all $2^{26}$ possible IO sets it is clear that they are feasible or not. Each vertical sequence is thus a trace of evaluated IO sets which ends in finding a minimal dependent set. For this case the number of evaluations is 815 
and $M=108$.

Figure 8 presents results for finding maximal independent sets, employing a bottom-up search direction. Here we start Traces of tested combinations

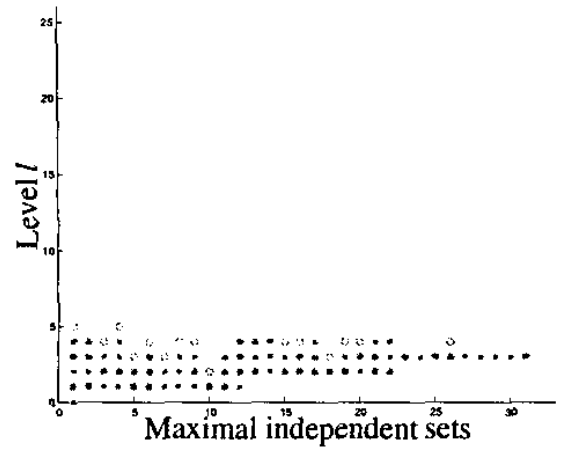

Figure 8: Search for maximal independent input sets for $\gamma=$ 1.5: " $o$ ": feasible ones, " $x$ ": infeasible one

with the empty IO set (the open loop), which is not feasible, and add devices until no device can be added without the resulting IO set becoming feasible. The number of evaluations is 348 and $K=31$, so this search direction needs less evaluations.

The combinatorial part of the search is in guaranteeing that IO sets are not evaluated when from the available results it is clear that they are feasible or infeasible. Details of how this can be done efficiently are in [7]. This part is easier for small values of $K$ and $M$. In general $K$ and $M$ are relatively small when either the specs are very tight (needing almost any device) or very loose (only a few devices are needed).

Combining the most promising actuators with similar results for sensor selection gives a selection problem with 8 input and 24 output devices, so $n=32$. In this case we do not use an equal number of sensors and actuators in the base set, because more sensors are needed than actuators to achieve a desired level of performance, as will become clear from the results presented. The selection is solved for $\gamma=1$, so only slightly worse than achievable with the full set of devices. The most promising devices are indicated with large bars in Fig. 9, because they appear in all 136 minimal dependent sets. The 8 actuators have device numbers $1-8$. Note that the

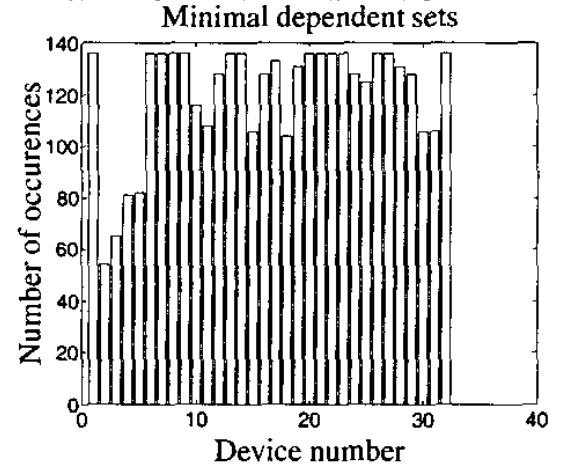

Figure 9: Results for minimal dependent IO sets for $\gamma=1$ number of sensors needed is much larger than the number of actuators. This is due to the relatively large weighting for the measurement noise. It shows that a restriction for actuators and sensors to appear as colocated pairs would require a larger number of devices to achieve the same performance.
A physical interpretation of the results indicates that actuators and sensors connected and "parallel" to the disturbance are selected, and devices not directly connected to the disturbance are eliminated. Note that these results differ from the results for dynamic stiffness. With the different goals targeted by the control specifications, this is not surprising.

Given the straightforward physical interpretation of the results, we do not expect a search based on all 52 devices to give results that are significantly different from the results obtained by a staged approach (first input and output selection separately, then a combined IO selection).

\section{Conclusions}

An efficient method for input/output selection was shown to be readily applicable to tensegrity structures with a larger number of potential IO sets. Modifications with minor consequences were needed to deal with the relatively large number of devices from which to choose.

The trend revealed for vibration reduction problems is to choose actuators in strings parallel to the disturbance vector, whereas, for dynamic stiffness improvement, the best actuator strings are perpendicular to the disturbance vector. The same holds for sensors. This shows that the set of feasible solutions depends largely on the goal of the controlled system.

The results are also beneficial when making choices in the design of tensegrity systems, because they indicate which tendons are needed and which can be eliminated when there is some redundancy in the tendons, i.e., in case not all of the original tendons are needed to assure integrity.

\section{References}

[1] R. Adhikari, R. Skelton, and W. Helton, "Mechanics of tenseg. rity structures," Feb. 1998. Report 1998-01 UCSD Structural Systems and Control Lab.

[2] J. C. Maxwell, The Scientific Papers of James Clerk Maxwell. New York: Dover, Forew. 1890.

[3] R. Skelton, "An introduction to controllable tensegrity structures," in Proc. 12th ASCE Engineering Mechanics Conf., May 1998. [4] M. van de Wal and B. de Jager, "A review of methods for input/output selection," Automatica-J. IFAC, vol. 37, pp. 487-510, Apr. 2001.

[5] B. de Jager and $O$. Toker, "Complexity of input output selection," in Proc. of the Intemat. Symp. on the Mathematical Theory of Networks and Systems (MTNS 98) (A. Beghi, L. Finesso, and G. Picci, eds.), (Padova, Italy), pp. 597-600, Il Polygrafo, July 1998. [6] B. de Jager and M. van de Wal, "Efficient selection of inputs and outputs for robust control," in Proc. of the 38th IEEE Conf. on Decision and Control, (Phoenix, AZ), pp. 4517-4522, IEEE, Piscataway, NJ, Dec. 1999.

[7] B. de Jager and J. Banens, "VISOR: Vast independence system optimization routine," Algorithmica, 2001. To appear.

[8] E. L. Lawler, J. K. Lenstra, and A. H. G. Rinnooy Kan, "Generating all maximal independent sets: NP-hardness and polynomialtime algorithms," SIAM J. Comput., vol. 9, pp. 558-565, Aug. 1980. [9] K. Glover and J. C. Doyle, "State-space formulae for all stabilizing controllers that satisfy an $H_{\infty}$ norm bound and relations to risk sensitivity," Systems Control Lett., vol. 11, pp. 167-172, 1988. [10] T. Iwasaki and R. E. Skelton, "All controllers for the general $\mathcal{H}_{\infty}$ control problem: LMI existence conditions and state space formula," Automatica-J. IFAC, vol. 30, pp. 1307-1317, Aug. 1994.

[11] P. Gahinet and P. Apkarian, "A linear matrix inequality approach to $\mathcal{H}_{\infty}$ control," Internat. J. Robust Nonl. Control, vol. 4, pp. 421448, Jan.-Feb. 1994. 